\title{
Variable Response to a Candidate Cancer Vaccine Antigen: MHC Control of the Antibody Response in the Rat to Avian Erythroblastosis Virus (AEV)- Encoded Epithelial Growth Factor Receptor but Not AEV-Encoded Thyroid Hormones Receptor
}

\author{
Nance Nardi ${ }^{1}$ and Nicholas A. Mitchison \\ Imperial Cancer Research Fund Tumor Immunology Unit, University \\ College London, London, United Kingdom
}

\begin{abstract}
Background: A problem likely to be encountered in any cancer immunotherapy based on vaccination with a single protein or peptide is variation in the host response. A particularly informative example is provided by the two oncogenic proteins, one intracellular and the other extracellular, encoded by the avian erythroblastosis virus (AEV), homologs of the thyroid hormones receptor (THsR) and the epithelial growth factor receptor (EGFR), respectively.

Materials and Methods: Antibodies to these two proteins were assayed by radioimmune precipitation (RIP) in sera from MHC-congenic rats immunized by virally induced tumors.

Results: Among the four haplotypes tested, $\mathrm{RTl}^{1}$ rats exhibited a significantly lower response to the EGFR homolog than the high responders $\mathrm{RTI}^{\mathrm{c}}$ and $\mathrm{RTl}^{\mathrm{u}}$, while
\end{abstract}

$\mathrm{RTl}^{\mathrm{a}}$ rat strains had an intermediate response. Analysis of the recombinant haplotype $\mathrm{RTl}^{\text {ac }}$ indicated that the response is controlled, as expected, by the class II locus of the MHC. In contrast, these rat strains responded uniformly to the intracellular THsR homolog.

Conclusions: These results support the hypothesis that MHC restriction of the response to self-related proteins reflects mainly a tolerance mechanism. They sound a note of warning for cancer vaccine development, and also one of positive advice. The likelihood of MHC restriction suggests that a widely applicable polyvalent vaccine should be the final aim in cancer immunotherapy. Yet, paradoxically, evidence of MHC restriction can help establish that a candidate vaccine is likely to prove effective.

\section{INTRODUCTION}

Hope is growing for cancer immunotherapy in general $(1,2)$ and for that involving the epithelial growth factor (EGF) receptor (3-5) in particular. This glycoprotein, which is encoded by the c-erb B gene, is a particularly attractive target because

Address correspondence and reprint requests to Nicholas A. Mitchison at his present address: Deutsches Rheuma-Forschungszentrum, Nordufer 20, 13353 Berlin, Germany.

'Present address: Universidade Federal del Rio Grande do Sul, Laboratorio de Imunogenetica, Avenida Bento Goncalves, 9500, Porto Alegre, Rio Grande do Sul, CEP90.000 BRAZIL. it is exposed on the cell surface and is overexpressed in certain tumors (6). As has been experienced with other single-protein or single-peptide vaccines $(7,8)$, some individuals are likely to be unable to respond, and this will be due in part to the presence of nonresponder MHC alleles.

A well-established animal model for examining this possibility is the rat. Neonatal rats injected with fibroblasts transformed by avian erythroblastosis virus (AEV) develop tumors at the site of innoculation in $50-60 \%$ of the animals within 6-8 weeks (9). F1 Fisher $\times$ Lewis hybrid rats produce antibodies against the 
v-p75 gag-erb A and v-p66/68 ${ }^{\text {erb B }}$ proteins. The erb A protein is a mutated version of the chicken high-affinity receptor for thyroid hormones, located in the nucleus (10), while the erb B protein is a version of the chicken EGF receptor. Of importance to the present study, it was noted that Fisher rats produce small amounts of antierb B antibodies (M. J. Hayman, personal communication). Because high-responder MHC alleles are normally dominant to low-responder ones, an experiment was performed in which the low-responder Fisher MHC was combined with various other MHC haplotypes, all as Fl hybrids with the same genetic background. An additional advantage of this system is that the coexpression of the intracellular erb A and cell-surface erb B proteins allows for these two types of antigens to be compared in terms of MHC restriction.

\section{MATERIALS AND METHODS}

The animals used are listed in Table 1, together with their origins and allele assignments at the major histocompatibility loci RG1.A and RG1.B (11). The MHC-congenic PVG strains were purchased from Olac (London, United Kingdom), and the F334 strain of Fisher rat obtained from the Imperial Cancer Research Fund breeding colony.

Tumor induction and the preparation of antiserum were as described (9). Clone ATla of Fisher fibroblasts transformed by avian erythroblastosis virus (AEV) were innoculated intraperitoneally $\left(5 \times 10^{6}\right.$ cells $)$ into 7 - to 14-day-old Fisher $\times$ Lewis Fl hybrid rats. The animals that developed tumors were bled by cardiac puncture 8-12 weeks after the innoculation.

Individual sera were analyzed by radioim-

TABLE 1. Alleles at the major histocompatibility complex of the rat strains used

\begin{tabular}{lccc}
\hline Strain & $\begin{array}{c}\text { Origin of } \\
\text { Haplotype }\end{array}$ & $\begin{array}{c}\text { RT1.A } \\
\text { (class I) }\end{array}$ & $\begin{array}{c}\text { RT1.B } \\
\text { (class II) }\end{array}$ \\
\hline F344 & F344 & $\mathrm{l}$ & $\mathrm{l}$ \\
PVG & PVG & $\mathrm{c}$ & $\mathrm{c}$ \\
PVG.RT1 $^{\mathrm{u}}$ & AO & $\mathrm{u}$ & $\mathrm{u}$ \\
PVG.RT1 $^{\text {rl }}$ & rl & $\mathrm{a}$ & $\mathrm{c}$ \\
PVG.RT1 $^{1}$ & AS & $\mathrm{l}$ & $\mathrm{l}$ \\
PVG.RT1 $^{\text {a }}$ & $\mathrm{DA}$ & $\mathrm{a}$ & $\mathrm{a}$ \\
& & & \\
\hline
\end{tabular}

mune precipitation on AEV-transformed chicken erythroblasts, according to methods previously described (9). The gels were scanned on a double-beam microdensitometer (Joyce-Loebl MkIII, Newcastle, United Kingdom). Relative band density was calculated in comparison with a standard positive antiserum which was included in each gel. Readings were taken from the $\mathrm{p} 75$ band of erb A and the combined p66 plus p68 bands of $e r b \mathrm{~B}$.

\section{RESULTS}

A representative autoradiograph of radioimmune precipitates prepared from 14 individual sera, together with positive and negative controls, is shown in Fig. 1. All the rats produced approximately the same amount of anti-erb A (p75) antibody. The levels of anti-erb B antibody are clearly different, with one group making high levels of antibody and the other (sera 11-14) little or none. While the resolution of erb $\mathrm{A}$ and erb B is good, that of the two erb B (p66 and p68) components is less clear. For this reason, measurements of the these two components were pooled.

Figure 2 shows the measured levels of antibodies. Measurements did not vary significantly between groups for the anti-erb A antibody, but clearly did so for the anti-erb B antibody. The first group of sera, from $\mathrm{RTl}^{1 / \mathrm{c}}$ rats, was taken as standard, from which the second, third, and sixth groups $\left(\mathrm{RT}^{1 / \mathrm{u}}, \mathrm{RT} 1^{1 / \mathrm{rl}}\right.$, and $\mathrm{RTl}^{\mathrm{a} / \mathrm{l}}$, respectively) did not differ significantly. The fourth group $\left(\mathrm{RTl}^{1 / 1}\right)$ was significantly lower $(t=4.57$, $p<0.0001)$; as the standard deviations of the two groups were significantly different, a nonparametric statistical analysis was also applied (Mann-Whitney $p=0.0005$ ). The fifth group $\left(\mathrm{RT}^{1 / a}\right)$ was also significantly lower $(t=1.89$, $p=0.032$ ), but, nevertheless, still significantly higher than the fourth group $(t=3.36, p=$ 0.002).

The fifth group $\left(\mathrm{RTI}^{1 / a}\right)$ was significantly lower $(t=2.10, p=0.040)$ than the third group $\left(\mathrm{RGl}^{1 / \mathrm{rl}}\right)$. This comparison is a particularly important because it provides information on the role of the class II RGI.B locus in the control of this response. These two groups differ at RGl.B but not at RGl.A, suggesting that the substitution of $R G 1 . B^{a}$ for $R G l . B^{c}$ is responsible for this reduction. We therefore provisionally identify this allele as intermediate in its effect. However, the reciprocal hybrids $\left(\mathrm{RTI}^{\mathrm{a} / \mathrm{l}}\right.$, sixth group) 


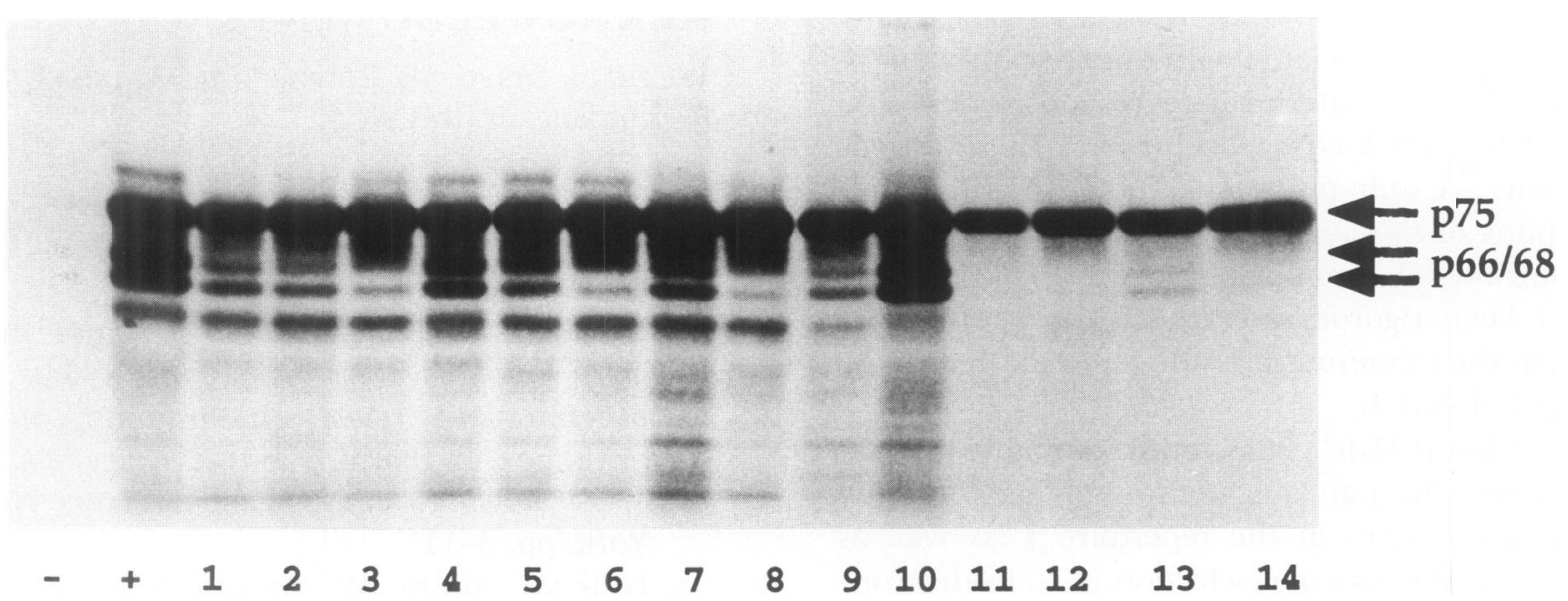

FIG. 1. Radioimmune precipitation of erb A (p75) and erb B (p66/68)

Lane - contains normal serum; Lane +, standard positive antiserum; Lanes 1-14, sera from individual rats developing ascitic or solid tumor derived from AEV-transformed rat fibroblasts. All sera contain antibodies to erb A; Sera 1-10 contain antibodies to erb B; Sera 11, 12, and 14 are negative for these antibodies, and Serum 13 is a weak positive (relative band density $<0.1$ ).

showed a lesser reduction, casting some doubt on this conclusion. Furthermore, these data do not exclude possible effects mediated by class III loci (for instance the TNF genes), which in the rat are located to the right of class II.

From the vaccine angle, an important question is how often does this form of immunization entirely fail to elicit an effective antibody response. A relative band intensity of $<0.1$ was given by 5/46 (11\%) high responders (Groups 1, 2 , and 3), 4/21 (19\%) intermediate responders
(Group 5), and $11 / 27$ (41\%) low responders (Group 4). The frequency of these low-to-zero responses thus runs parallel with the mean levels of response.

\section{DISCUSSION}

These data clearly show that ability to respond to the erb B viral-oncogene product segregates with the MHC in these congenic rats, probably with

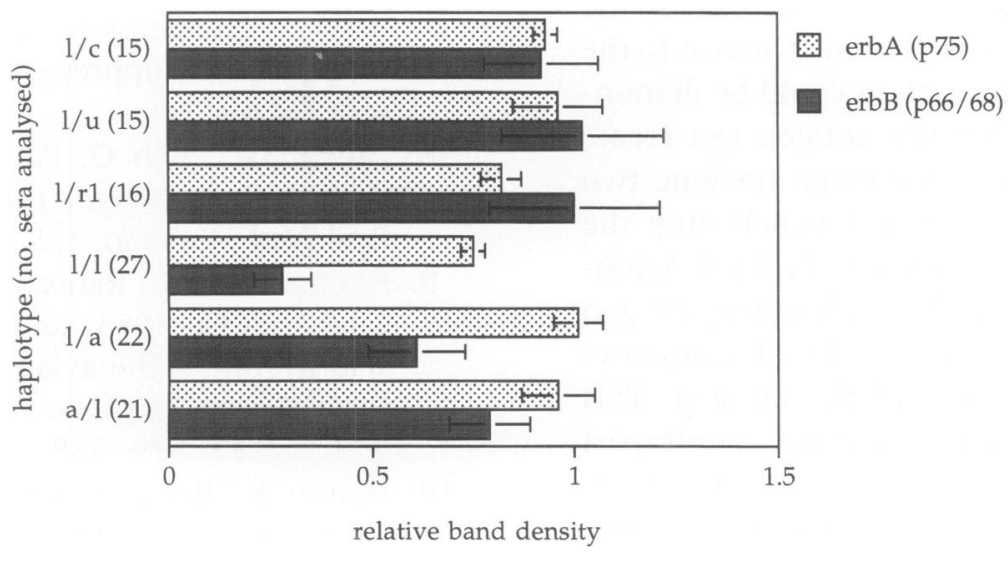

FIG. 2. Quantitative analysis of sera from rat F1 hybrids between F344 and five RT1-congenic PVG strains

In the haplotype designations the female parent is given first (e.g., the designation l/c indicates an Fl hybrid offspring of an $\mathrm{RGI}^{1}$ female and an $\mathrm{RGI}^{\mathrm{c}}$ male). In the first five groups the female parent was $\mathrm{F} 344$, except in the last group where the female parent was PVG-RT1 ${ }^{\mathrm{a}}$. The number of sera (collected from individual rats) is shown in parenthesis. Means and standard errors are shown. 
the RG1.B class II locus. The alleles RGI. $B^{c}$ and $R G 1 . B^{u}$ are associated with high responsiveness, RGI.B ${ }^{a}$ with intermediate responsiveness and RGI.B ${ }^{1}$ with low responsiveness. These assignments are only provisional, because of the limitations of this type of genetic analysis; possible contributions from genes outside the MHC have not been rigorously excluded, nor have those from the numerous class III genes located to the right of RGI.B.

Class II MHC genes control immune responsiveness through mechanisms of tolerance (by creating "holes in the repertoire"), as well as through determinant selection (12). In this context, it is of interest that the response to erb B but not to erb A is under class II polymorphic control. Why determinant selection should make such a distinction is not obvious. On the other hand, one would expect a cell-surface (and body fluid) protein, such as the erb B product, to have better access to Th cells than an intracellular protein, such as that encoded by erb A, and therefore to be better able to operate a mechanism of tolerance (13). Furthermore, the viral oncogenes which encode the erb A and $e r b$ B proteins have proto-oncogene homologues in the host, making a tolerance mechanism of MHC-mediated unresponsiveness all the more likely. A critical experiment would be to test whether the erb B T-cell epitope binds to the nonresponder-type MHC molecule; the tolerance hypothesis predicts that the epitope would bind but not stimulate $\mathrm{T}$ cells.

This last point can be expanded. To provide an effective vaccination target, a cancer cell antigen needs to be accessible to Th cells. If the argument proposed above is correct, access to the Th cells of the immune system could be demonstrated by showing that the antigen can create holes in the repertoire. One could imagine two candidate vaccine antigens for which only the following properties are known: antigen A provokes a response in all individuals tested; antigen $B$ has nonresponders whose HLA molecules nevertheless prove able to bind the antigen. This information tells us that the normal counterpart of antigen $B$ is likely to have access to the immune system, while, in this respect, nothing would be known about antigen A. As a result, antigen $B$ could be the better choice. Thus, paradoxically, there are circumstances in which it may be better to choose a vaccine antigen with a variable response, in preference to one which behaves more uniformly.

\section{ACKNOWLEDGMENTS}

We thank Drs. G. W. Butcher and M. J. Hayman for guidance in this work.

\section{REFERENCES}

1. Urban JL, Schreiber H. (1992) Tumor antigens. Annu. Rev. Immunol. 10: 617-644.

2. Mitchison NA. (1994) The immunogenicity of tumors. In: Forni G (ed). Cytokine-Induced Tumor Immunogenicity. Academic Press, New York, pp. 3-11.

3. Disis ML, Smith JW, Murphy AE, Chen W, Cheever MA. (1994) In vitro generation of human cytolytic T-cells specific for peptides derived from the HUER-2/neu protooncogene protein. Cancer Res. 54: 1071-1076.

4. Ishida $T$, Tsujisaki $M$, Hanzawa $Y$, et al. (1994) Significance of erbB-2 gene product as a target for cancer therapy. Scand. J. Immunol. 39: 459-466.

5. Natali PG, Nicotra MR, Digiesi G, et al. (1994) Expression of gp 185HER-2 in human cutaneous melanoma: Implications for experimental immunotherapeutics. Int. J. Cancer 56: 341-346.

6. Libermann TA, Nusbaum HR, Razon N, et al. (1985) Amplification, enhanced expression and possible rearrangement of EGF receptor gene in primary human brain tumours of glial origin. Nature 313: 144-147.

7. Watanabe $H$, Okumura $M$, Hirayama $K$, Sasazuki T. (1990) HLA-Bw54-DR4-DRw53DQw4 haplotype controls nonresponsiveness to hepatitis- $B$ surface antigen via CD8-positive suppressor T cells. Tissue Antigens 36: 69-74.

8. Talwar GP, Sigh O, Pal R, et al. (1994) A vaccine that prevents pregnancy in women. Proc. Natl. Acad. Sci. U.S.A. 91: 8532-8536.

9. Hayman MJ, Ramsay GM, Savin K, Kitchener G. (1983) Identification and characterization of the avian erythrobalstosis virus gene product as a membrane glycoprotein. Cell 32: $579-588$.

10. Damm K, Beug $H$, Graf $T$, Vennstrom B. (1987) A single point mutation in erbA restores the erythroid transforming potential of a mutant avian erythroblastosis virus (AEV) defective in both erbA and erbB oncogenes. E.M.B.O. J. 6: 375-382.

11. Cortese Hassett AL, Misra DN, Kunz HW, Gill III TJ. (1991) The major histocompati- 
bility complex of the rat. In: Srivastava $\mathrm{R}$, Ram BP, Tyle P (eds). Immunogenetics of the Major Histocompatibility Complex. VCH publishers, New York, pp. 309-347.

12. Sette A, Sidney J, Gaeta FC, et al. (1993) MHC class II molecules bind indiscriminately self and non-self peptide homologs: Effect on the immunogenicity of non-self peptides. Int. Immunol. 5: 631-638.

13. Salvat S, Auger I, Rochelle L, et al. (1994) Tolerance of a self-peptide from the third hypervariable region of HLA DRB 1 * 0401 in rheumatoid arthritis patients and normal subjects. J. Immunol. 153: 5321-5329.

Contributed by N. A. Mitchison on April 17, 1995. 